\title{
A Case of Prurigo Nodularis Treating by Acupuncture
}

\author{
Dan $\mathrm{Wu}^{\prime}$ \\ Guirong Dong ${ }^{2}$ \\ 'Shanghai Academy of Traditional \\ Chinese Medicine, Shanghai, 200I20, \\ People's Republic of China; ${ }^{2}$ Department \\ of Acupuncture, Yueyang Hospital of \\ Integrated Traditional Chinese and \\ Western Medicine, Shanghai University of \\ Traditional Chinese Medicine, Shanghai, \\ 20000, People's Republic of China
}

\begin{abstract}
Aim: Male, 68 years old, suffering from prurigo nodularis for 7 months, and did not show any significant improvement after oral and topical medications. After 4 months of acupuncture treatment, the NRS for itch scores decreased from 9 to 1; the NRS for sleep scores increased from 5 to 9; HADS decreased from 14 to 2; DLQI decreased from 9 to 5; IGA decreased from 4 to 3. After a 2-month follow-up, the patient was stable in all aspects without new rash.
\end{abstract}

Conclusion: Acupuncture is a feasible method for the treatment of prurigo nodularis.

Keywords: prurigo nodularis, acupuncture, acupoints

A 68-year-old male patient suffered from prurigo nodularis for 7 months. No significant improvement was observed after 3 months of oral antihistamine drugs such as Cetirizine Hydrochloride Tablets (1 tablet Qd), Ketotifen Fumarate Tablets (1 tablet Qd), and topical hormone creams such as Halometasone Cream (Bid), Desonide Cream (Bid) in local hospitals. The patient has no history of atopic dermatitis and benign prostate tumors for 3 years. Physical examination revealed widespread hyperkeratosis nodules and excoriations on scalp, extensor side of the extremities, and trunk (Figure 1).

The Numerical Rating Scale (NRS) for itch expresses 9 points ( 0 means no pruritus, 10 means worst pruritus), patients say the itching is uncontrollable and frightening. The NRS for sleep scores 5 points ( $0-10$ points, higher scores manifest worse sleep quality), the Hospital Anxiety and Depression Scale (HADS) scores 14 points (HADS-A scores 8 points, and there was a clear anxiety tendency), the Dermatology Life Quality Index (DLQI) scores 9 points, and the Investigator's Global Assessment Scale (IGA) scores 4 points. The blood IgE level is $365 \mathrm{IU} / \mathrm{mL}$ (normal range $<100.0 \mathrm{IU} / \mathrm{mL}$ ). We state that consent to publish the case details and any accompanying images has been obtained from the patient. At the same time, the patient gave informed consent to the publication of case details and images.

Acupoints selection: Baihui (GV20), Shenting (DU24), Fengfu (DU16), Daizhui (DU14), Zhongwan (CV12), Qihai (RN6), Bilateral Fengchi (GB20), Geshu (BL17), Tianshu (ST25), Xuehai (SP10), Zusanli (ST36), Sanyinjiao (SP6), Xuqu (GB40), Chongyang (ST42), Jinggu (BL64), Quchi (LI11), Waiguan (SJ5), Hegu (LI4), Yangchi (SJ4), Wangu (SI4).

Manipulation: Routine sterilization, disposable sterile filiform needle, $0.25 \times 40 \mathrm{~mm}$ was selected. For GV20, DU24, insert needles perpendicularly and going beneath the epicranial aponeurosis, then change the angle into $15^{\circ}$ against scalp, 20-30mm in depth. At DU16, GB20, pierce the needle $20-30 \mathrm{~mm}$ towards mandible. At DU14, BL17, insert needles obliquely with an angle of $15^{\circ}, 20-30 \mathrm{~mm}$ in depth. Keep the needles on and ask the patient lay in supine position, at CV12,
Correspondence: Guirong Dong Tel +86-18930132113

Email dongguirong2000@126.com 

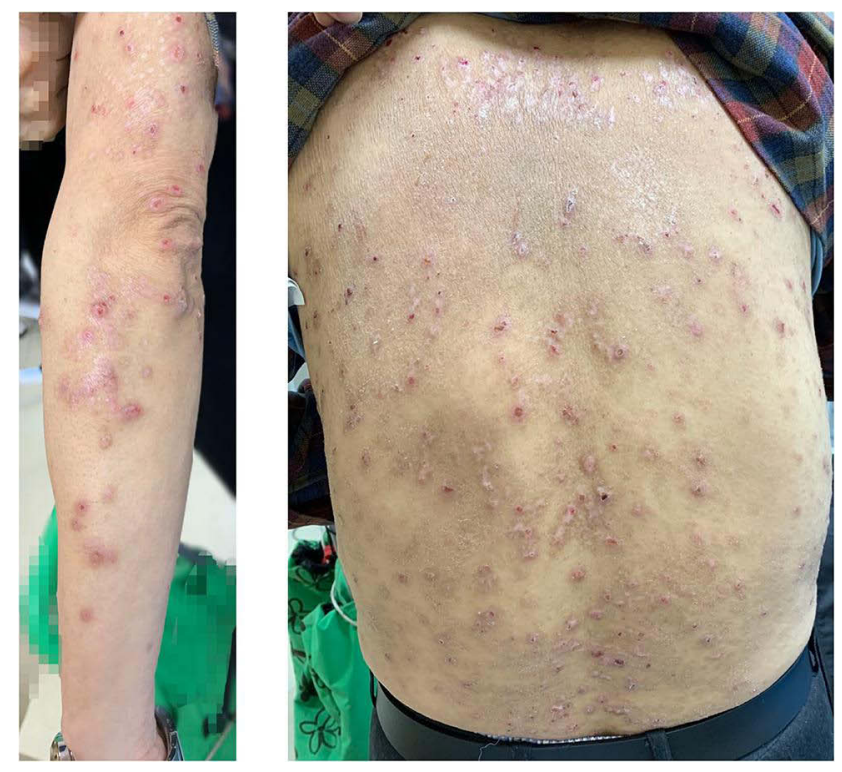

Figure I W0 (prurigo nodularis lesions at baseline).

ST25, RN6, LI11, SJ5, LI4, SJ4, SI4, SP10, ST36, SP6, GB40, ST42, BL64, the needle are inserted perpendicularly, $25-35 \mathrm{~mm}$ in depth. All the needles are retained for $30 \mathrm{~min}$. The treatment was given three times a week.

In the second week of acupuncture treatment, the itch NRS scored 7 points. There were few new rashes. After 4 weeks of treatment, the itch NRS scored 4 points. The skin damage was significantly improved, and some small nodules were flattened. No new rash (Figure 2). The NRS for sleep scored 8 points. In the 8th week, due to overwork in home decoration, the score of itch NRS was 6 . No new rash; in the 10th week, majority of nodules are flattened, except the dozens of most big ones. The NRS for itch scored 2 points, indicating that it is basically not itchy during the day, and slight itching at night before bedtime, which could be controlled without scratching (Figure 3). At week 13, the itch NRS scored 2 points and had fewer rashes. In the 16th week, all but a small number of nodules on both upper limbs had subsided with pigmentation left (Figure 4). The itch NRS scored 1 point, the NRS for sleep scored 9 points, the HADS scored 2 points, the DLQI scored 5 points, and the IGA scored 3 points. The blood $\mathrm{IgE}$ level is $41 \mathrm{IU} / \mathrm{mL}$ (normal range $<100.0 \mathrm{IU} / \mathrm{mL}$ ). No discomfort occurred during the treatment. During the 2-month's follow-up, the patient was stable in all aspects without new rash (Figures 5 and 6).

Prurigo nodularis is an intractable pruritus skin disease that is prone to occur on the extensor aspect. Cause of the
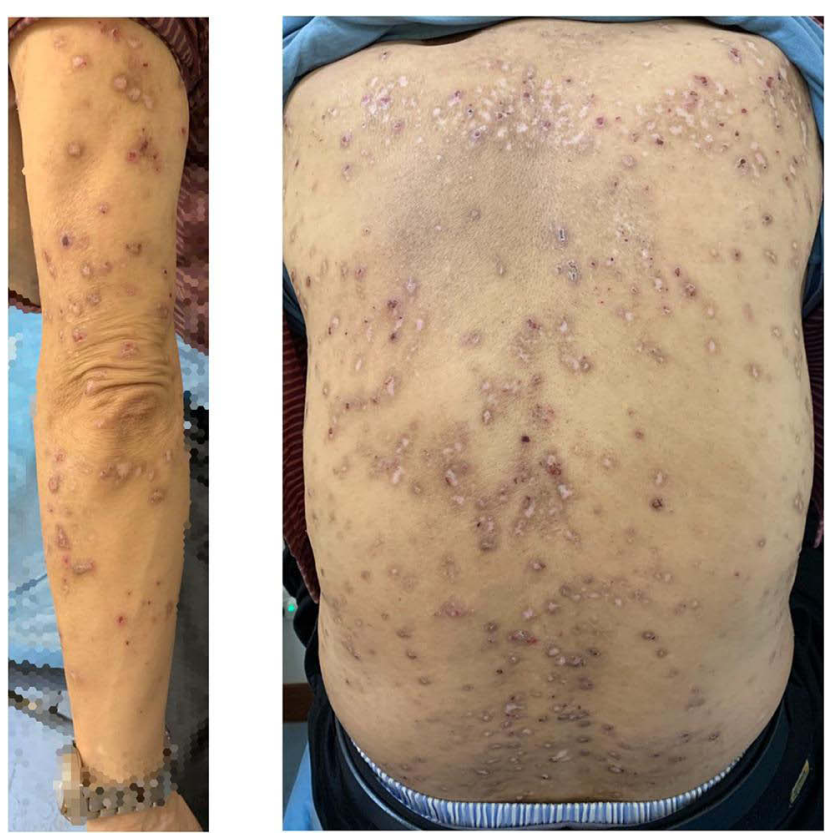

Figure 2 W4 (prurigo nodularis improved after acupuncture therapy at week 4).
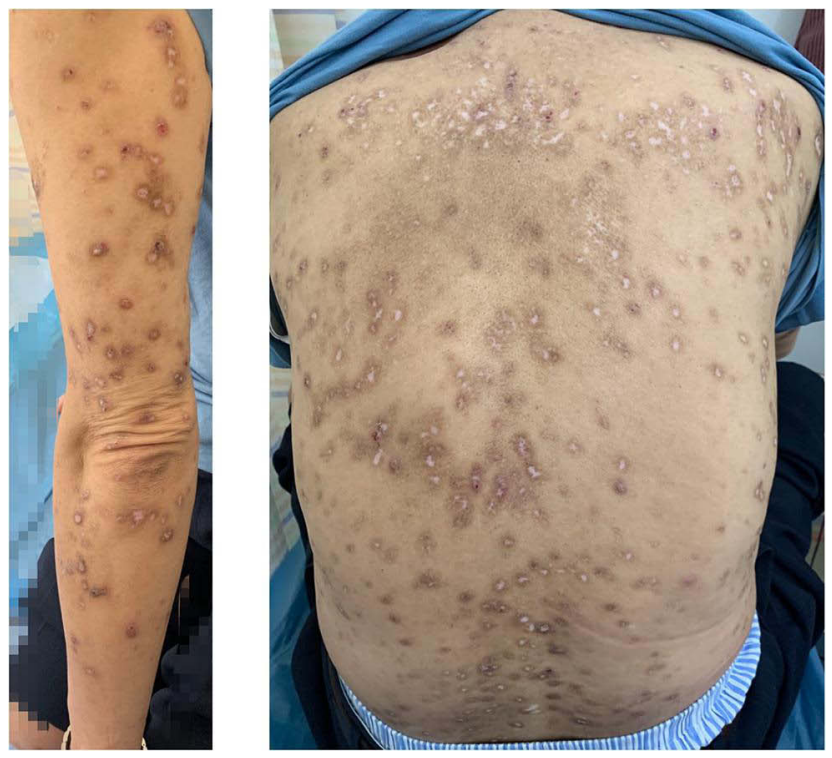

Figure 3 WIO (prurigo nodularis improved after acupuncture therapy at week 10).

disease is not yet clear. Compared with other skin diseases such as atopic dermatitis and psoriasis, $\mathrm{PN}$ is more likely to be associated with depression or anxiety. ${ }^{1}$ Conventional treatment of oral antihistamine drugs, immunosuppressants, etc., have different degrees of side effects. Biologics such as Dupilumab and Nemolizumab have been proved to be effective, ${ }^{2,3}$ but the new drugs have disadvantages such as expensive price and long service cycle. External treatment of traditional Chinese medicine such as fire needle has been 

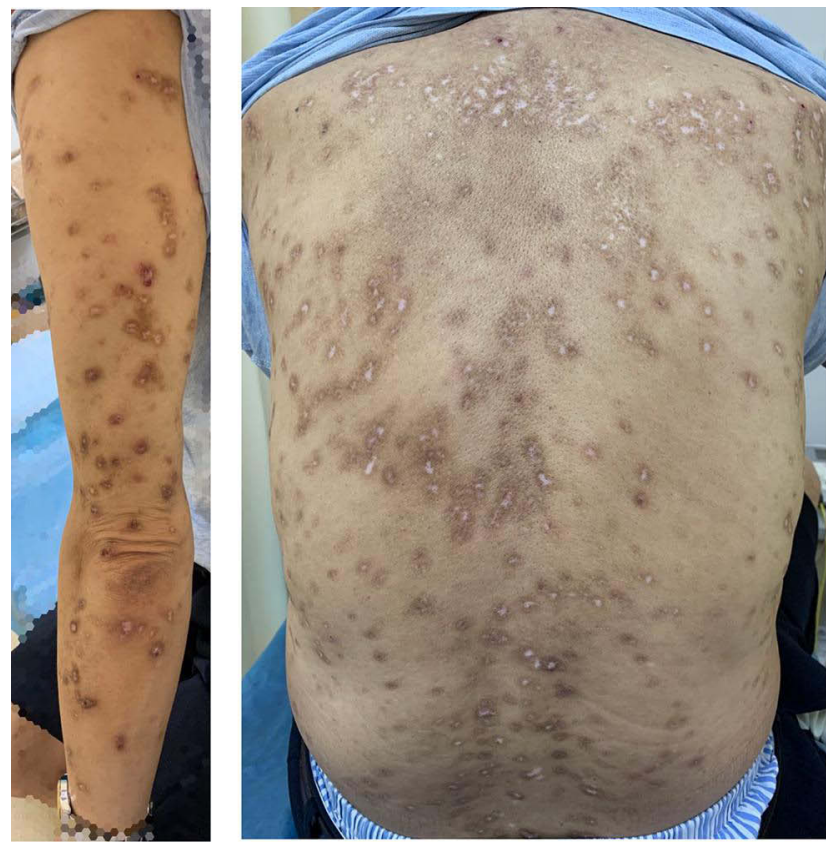

Figure 4 WI6 (prurigo nodularis improved after acupuncture therapy at week 16).
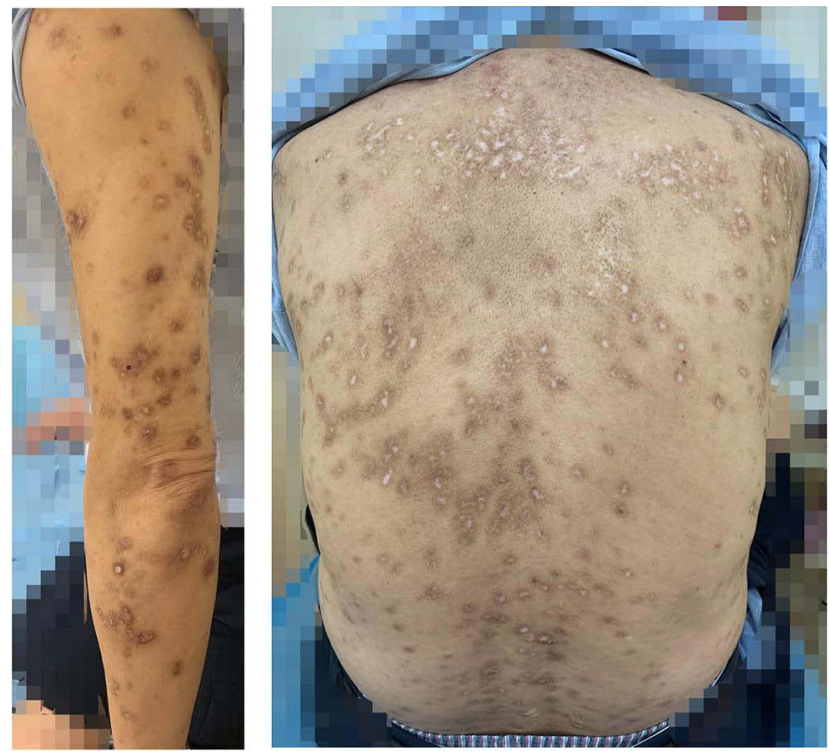

Figure $5 \mathrm{FI}$ (followed up for I month).

proved to have a certain effect, ${ }^{4}$ but the operation is complicated for patients with more rashes. Chinese acupuncture, with its characteristics of regulating Yin and Yang, dredging channels and collaterals, calming nerves and thoughts, strengthening Qi and resisting evil, thus, can help patients with nodular pruritus to improve sleep quality, regulate bad emotions, and then promote the regression of rash, so that to forms a benign cycle. Traditional Chinese medicine recognizes: "All pains are excess; all itches are deficiency. The
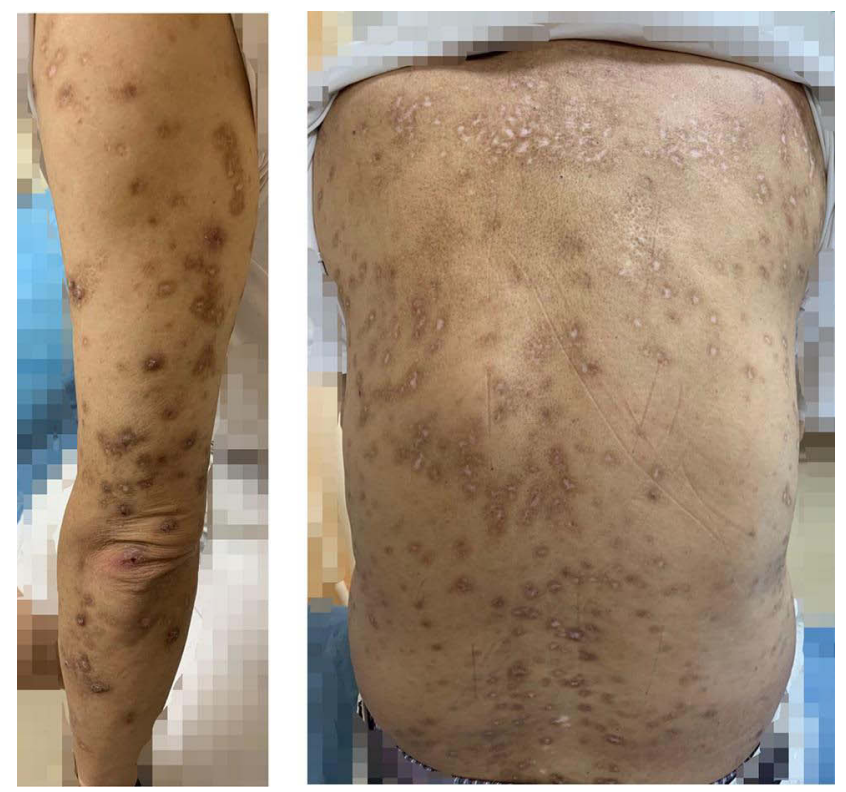

Figure 6 F2 (followed up for 2 month).

excess, the evil qi is real; the deficiency, the righteous qi is weak ... the one who often itch, this righteous qi is also weak." This suggests that acupuncture treatment should take vital Qi as prime. GV20, DU24, and DU16 can calm the nerves and promote the brain to play a leading role, which is the key point for improving emotions. CV12, ST25, RN6, and ST36 regulate the function of the spleen and stomach and protect righteous qi (the qi of the spleen and stomach is also called righteous qi). DU14, GB20, LI11, SJ5, BL17, SP10, SP6 can disperse wind, cool blood, activate blood and stop itching. Prurigo nodularis is prone to occur on the extensor aspect of the body, which, in TCM theory, is also part of the skin of Six Yang Meridians of hands and feet. GB40, ST42, BL64, LI4, SJ4, and SI4 are the original acupoints of the Six Yang Meridians of the hands and feet, which can stimulate the circulation of the energy of these Yang Meridians. Acupuncture has been proven to play a role of regulating nerves, immunity, endocrine. Some studies have shown that acupuncture may activate $\mathrm{C}$ fibers to consume neurotransmitters, inhibit mast cells to release a variety of itching mediators, etc. to play an antipruritic effect. ${ }^{5}$

Acupuncture therapy can quickly relieve the degree of pruritus, control new rashes, and improve patients' anxiety and depression, as well as sleep quality and life quality. From the follow-up results, it can be seen that the curative effect of acupuncture is lasting and stable. From this case we can tell that acupuncture therapy can greatly improve all aspects of discomfort in patients with prurigo nodularis. 
It has the advantages of one therapy with multiple effects, no side effects, and low economic burden.

\section{Ethical Approval}

This study was approved by the Ethics Committee of Shanghai Yueyang Hospital of Integrated Traditional Chinese and Western Medicine and was in accordance with the 1964 Helsinki declaration and its later amendments or comparable ethical standards. Written informed consent to participate was obtained from all of the individual participants included in the study.

\section{Disclosure}

The authors report no conflicts of interest in this work.

\section{References}

1. Huang AH, Williams KA, Kwatra SG. Prurigo nodularis: epidemiology and clinical features. $J$ Am Acad Dermatol. 2020;83(6):15591565. doi:10.1016/j.jaad.2020.04.183

2. Chiricozzi A, Maurelli M, Gori N, et al. Dupilumab improves clinical manifestations, symptoms, and quality of life in adult patients with chronic nodular prurigo. J Am Acad Dermatol. 2020;83(1):39-45. doi:10.1016/j.jaad.2020.03.049

3. Ständer S, Yosipovitch G, Legat FJ, et al. Trial of nemolizumab in moderate-to-severe prurigo nodularis. $N$ Engl $J$ Med. 2020;382 (8):706-716. doi:10.1056/NEJMoa1908316

4. Luo Y, Kuai L, Song N, et al. Efficacy and safety of fire needle therapy for nodular prurigo: a quantitative study. Evid Based Complement Alternat Med. 2019;2019:8797056. doi:10.1155/2019/8797056

5. Haomiao B, Yan L, Liuliu Z, et al. Regulation mechanism of itch. $J$ Neuroanat. 2020;36(06):679-684.

\section{Publish your work in this journal}

Clinical, Cosmetic and Investigational Dermatology is an international, peer-reviewed, open access, online journal that focuses on the latest clinical and experimental research in all aspects of skin disease and cosmetic interventions. This journal is indexed on CAS.
The manuscript management system is completely online and includes a very quick and fair peer-review system, which is all easy to use. Visit http://www.dovepress.com/testimonials.php to read real quotes from published authors. 